\title{
RELATIONSHIP BETWEEN THE GENOME PACKING IN THE BACTERIOPHAGE CAPSID AND THE KINETICS OF DNA EJECTION
}

\author{
M. DE FRUTOS \\ Institut de Biologie et Biochimie Moléculaire et Cellulaire \\ UMR CNRS 8619, Bât 430, Université Paris Sud \\ 91405 Orsay cedex, France \\ Marta.de-Frutos@u-psud.fr \\ A. LEFORESTIER* ${ }^{*}$ and F. LIVOLANT ${ }^{\dagger}$ \\ Laboratoire de Physique des Solides, UMR CNRS 8502 \\ Université Paris-Sud, Bât 510 \\ Orsay 91405, France \\ *Amelie.Leforestier@u-psud.fr \\ $\dagger$ Françoise.Livolant@u-psud.fr
}

Received 27 July 2013

Revised 20 September 2013

Published 31 October 2013

\begin{abstract}
We present a general survey of experimental and theoretical observations of DNA structure and in vitro ejection kinetics for different bacteriophage species. In some species, like T5, the ejection may present pauses and arrests that have not been detected in others species like Lambda. We propose hypotheses to explain such differences and we discuss how the experimental conditions may be important for their detection. Our work highlights the role of DNA organization inside the bacteriophage capsid on the stochastic and out of equilibrium nature of the ejection process.
\end{abstract}

Keywords: Bacteriophage; DNA structure; DNA ejection; kinetics; fluorescence microscopy; cryoTEM.

\section{Introduction}

In tailed bacteriophages, a double-stranded DNA molecule is compacted within a icosahedral protein capsid. One of the apex of the icosahedron is occupied by a connector, the portal protein complex, extended by the tail. To infect their host, these phages, unlike eukaryotic viruses, do not penetrate inside the bacteria; they inject their DNA after binding the tip of the tail to a specific receptor located 
in the host envelope. The protein capsid and tail remain outside of the infected cell. Phages then use the bacterial machinery to synthesize new DNA and proteins. The empty procapsid is first assembled, and a DNA chain internalized through the pore of the portal with the action of a molecular motor using ATP hydrolysis as an energy source. The tail is self-assembled independently and secondly attached to the capsid. Finally, neo-synthesized phages are released into the external medium after the bacterial cell has been lysed. The whole process corresponds to a lytic cycle.

Much attention has been devoted to the question of DNA encapsidation, organization and ejection during the last few years. ${ }^{1-6}$ The question is indeed stimulating if we consider that a DNA molecule, a few tens of micrometers long, highly negatively charged, is confined in a volume of a size comparable to the persistence length of the chain $(50 \mathrm{~nm})$ at a concentration of about $500 \mathrm{mg} / \mathrm{ml}$. Such concentrations are not only the highest encountered in vivo, but are higher than those obtained when the DNA is condensed in vitro in the presence of multivalent cations (polyamines, cobalt hexamine,...). Experimentally they can be reached only by strong dehydration. ${ }^{7}$ The forces involved in packaging DNA into the capsid to such concentrations have been estimated theoretically ${ }^{8}$ and measured experimentally ${ }^{9}$ as being of the order of 50 to $100 \mathrm{pN}$, which corresponds to pressures of the order of 50 to $100 \mathrm{~atm}$ inside the full capsid. It was hypothesized that this pressure is (at least partly) responsible for the ejection of the DNA outside of the capsid during infection. ${ }^{10,11}$

In this article, we intend to concentrate on the question of the organization of the DNA chain inside the capsid, when it is completely full and also when it is progressively emptied during the ejection process and to discuss how this organization is related to the kinetics of the ejection in vitro. We will not discuss here the in vivo transfer of DNA from the bacteriophage to the bacteria. ${ }^{12}$ In vivo and in vitro phenomena differ in many respects and it is likely that the cell contribution becomes predominant compared to the viral one. Our aim here is to consider the complete set of existing data concerning DNA organization and ejection and to reanalyze them in order to propose a global and coherent description of the involved mechanisms. We include experimental and theoretical results already published and some new unpublished data. We will first briefly review (1) what is known about the DNA organization in dense liquid crystalline phases of short DNA fragments, in the absence of confinement and (2) what is known about the organization of DNA inside the full capsid at different steps of the ejection. Then, we will consider the kinetics of DNA ejection and compare data collected on several bacteriophages. In the last part of the article, we intend to relate structural and kinetic data and to discuss similarities and differences between bacteriophages T5 and Lambda. We will finally consider in the discussion what are the specific structural and topological constraints imposed by the confinement of the DNA and discuss how these may differ from species to species despite comparable DNA concentrations. We will finally propose an hypothesis to explain why pauses during the ejection process have been found in T5 and not in Lambda. 


\section{Results}

\subsection{Experimental results}

2.1.1. The experimental system for bacteriophage structure and ejection studies

We present on Fig. 1 how the bacteriophage fixes its tail to the membrane receptor to induce the release of the DNA chain into the host (Fig. 1(a)). This process can be reproduced in solutions containing only the bacteriophages and the purified receptors (Fig. 1(b)). In our experiments we used the bacteriophage T5 and its receptor FhuA. ${ }^{13,14}$ Others have been using the bacteriophages/receptors Lambda/LamB, ${ }^{15,16}$ or SPP1/YueB. ${ }^{17}$ These simplified systems offer the interest to monitor precisely a set of experimental conditions (ionic concentrations, temperature, osmotic pressure for example) and to analyze their effect on DNA organization and ejection. We must keep in mind that these do not reproduce the conditions of bacterial infection since all ingredients of the cell machinery are absent.

\subsubsection{Structure of DNA in the bulk}

The bacteriophage genome is highly concentrated and confined inside the protein capsid. But what do we know about the structure of DNA at such high concentration?

The pioneer works of Robinson, ${ }^{18}$ Luzzati $^{19}$ and Lerman $^{20}$ revealed the liquid crystalline structure of concentrated solutions of short DNA fragments. Further studies explored in more details the nature and conditions to obtain such DNA liquid crystalline states with DNA fragments of defined length, close to the persistence length of the chain $(50 \mathrm{~nm})$, under a large variety of ionic conditions. ${ }^{21}$ In the presence of monovalent ions, the following sequence was observed: isotropic

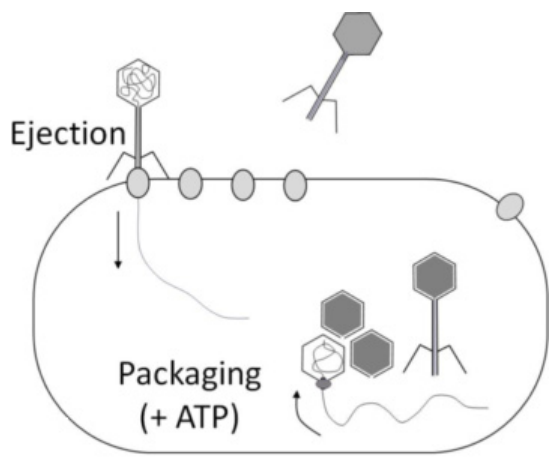

IN VIVO

(a)

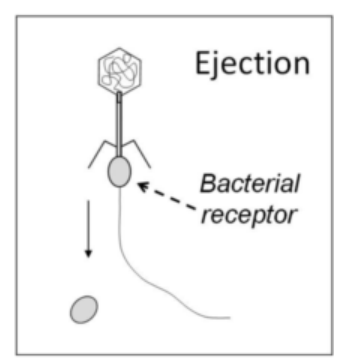

IN VITRO

(b)

Fig. 1. Synthetic representation of the process of DNA ejection and encapsidation occurring in vivo (a) and how the ejection process can be reproduced in vitro (b). 

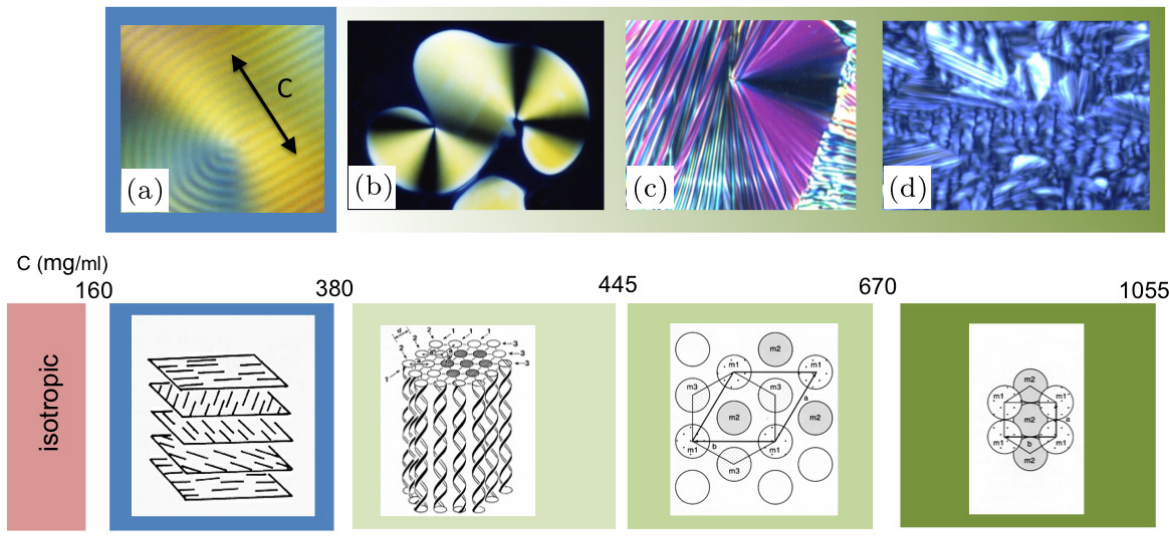

Interhelix

Distance 4.9

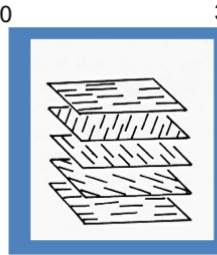

380

670

$(\mathrm{nm})$

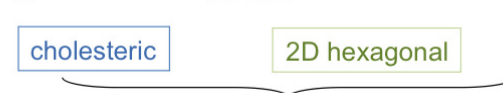

Liquid crystalline phases
445

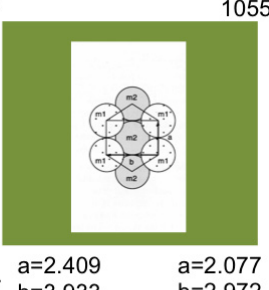

670

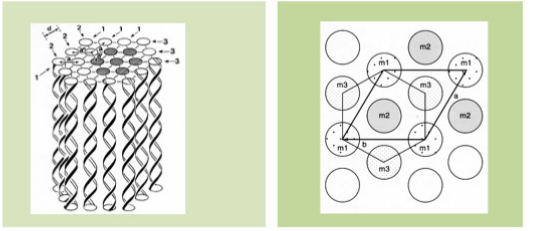

2.9

$2.37 b=3.933$

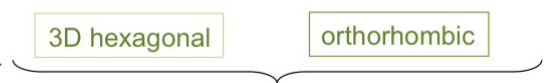

3D crystals

Fig. 2. (Color online) Concentrated phases of $50 \mathrm{~nm}$ DNA fragments observed in polarizing microscopy for increasing DNA concentrations: cholesteric phase (a), 2D columnar hexagonal phase (b) and 3D hexagonal and orthorhombic phases (c, d). The structure of each phase is schematized below, using the same color code. Concentrations (in $\mathrm{mg} / \mathrm{ml}$ ) and inter helix distances (in $\mathrm{nm}$ ) are indicated for each phase transition.

phase, blue phase (not illustrated here), cholesteric phase and 2D hexagonal phase (Fig. 2). For higher concentrations, the liquid crystalline solutions turn crystalline (3D hexagonal and orthorhombic). Interhelix distances at the phase transitions were determined by X-ray diffraction. ${ }^{7}$ They are given on Fig. 2 together with schematic drawings representing the organization of molecules in each phase. Similar phases were obtained later on with fragments of defined length and sequence. ${ }^{22,23}$

The cholesteric phase is characterized by fingerprint patterns when observed in polarizing microscopy (Fig. 2(a)) and by arched patterns drawn by DNA fragments of different orientations with the resolution of electron microscopy (Fig. 3(a $\left(\mathrm{a}^{\prime}\right)$ ). The stripes and arches, perpendicular to the cholesteric axis (indicated by $\mathrm{c}$ in Fig. $\left.2(\mathrm{a})^{\prime}\right)$, reveal the continuous rotation of the DNA direction as schematically drawn in Fig. 2(a) and Fig. 3(a). Each stripe corresponds to a rotation of $180^{\circ}$ of the director (half the helical pitch). The pitch is usually close to $2.5 \mu \mathrm{m}$ in monovalent salt. $^{21}$ This value may change significantly according to variations of the ionic conditions. It may go down to $0.25 \mu \mathrm{m}$ or up to $25 \mu \mathrm{m}$ (for example when multivalent cations spermidine are present ${ }^{24}$ ). In the $2 \mathrm{D}$ hexagonal phase, chains are aligned in parallel in a hexagonal lattice with no correlation from one column to the other. Textures are extremely bright with many rotation defects as seen on Fig. 2(b). Further on, textures turn less and less bright (Fig. 2(d)) as concentration increases due to the fractionation of the macroscopic domains into domains increasingly smaller and separated by defect walls. These walls include many twist 


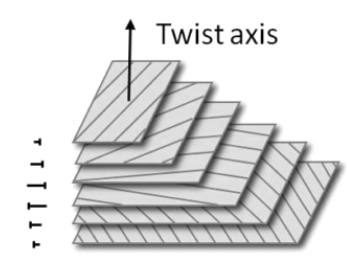

(a)

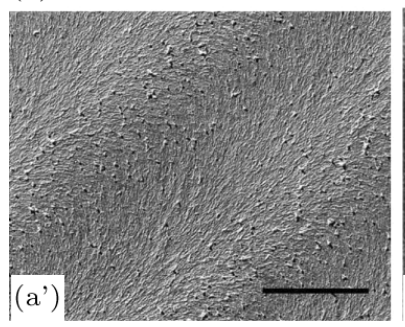

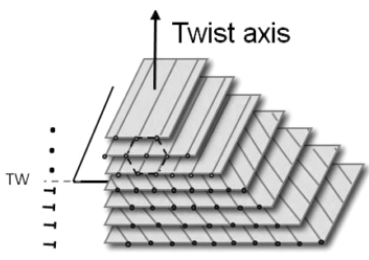

(b)

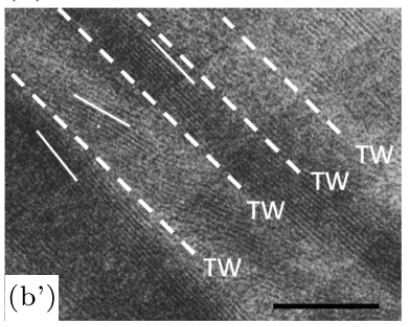

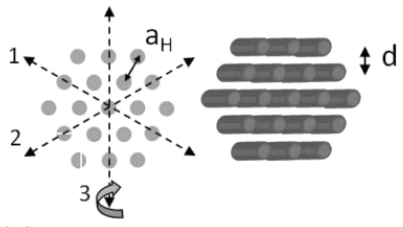

(c)

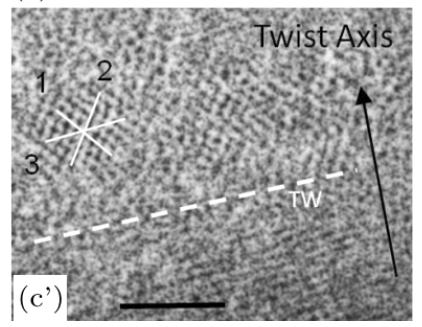

Fig. 3. Concentrated phases of DNA observed in electron microscopy. The cholesteric phase $\left(\mathrm{a}^{\prime}\right)$ reveals series of arched patterns drawn by the orientation of DNA fragments that rotate continuously along the cholesteric (twist) axis. In the $3 \mathrm{D}$ hexagonal phase $\left(\mathrm{b}^{\prime}, \mathrm{c}^{\prime}\right)$ the twist is located along twist walls (TW) separating domains of different orientations (underlined by white lines). The hexagonal lattice formed by the DNA helices may be seen at higher magnification under favourable orientation (top of the picture in $\left(c^{\prime}\right)$ ). It changes into striated patterns (of period $d=a_{H} \sqrt{3} / 2$, with $a_{H}$ the inter helix spacing) when the lattice is seen obliquely (bottom of the picture), as illustrated on the scheme above. In between is located the twist wall (parallel to DNA chains). Methods are respectively freeze-fracture in ( $\left.\mathrm{a}^{\prime}\right)$ (reproduced from Ref. 27) and cryosections of vitrified samples in $\left(\mathrm{b}^{\prime}, \mathrm{c}^{\prime}\right)$ (reproduced from Ref. 25). Scale bar $=3.5 \mu \mathrm{m}$ in $\left(\mathrm{a}^{\prime}\right)$, $50 \mathrm{~nm}$ in $\left(\mathrm{b}^{\prime}\right), 20 \mathrm{~nm}$ in $\left(\mathrm{c}^{\prime}\right)$.

walls revealed by freeze-fracture and cryoelectron microscopy of vitreous sections ${ }^{25}$ (Figs. 3( $\left.\mathrm{b}^{\prime}\right)$ and $3\left(\mathrm{c}^{\prime}\right)$ ). Briefly speaking, the phase turns $3 \mathrm{D}$ crystalline and can be considered as an equivalent of the TGB (Twist Grain Boundary) phase described in chiral liquid crystals. ${ }^{26}$ The twist arising from the chirality of the molecules (and initially present in the cholesteric DNA phase), is excluded from the 3D hexagonal phase and concentrated along the twist walls separating domains of different orientations. Instead of being continuous, the twist is now discrete and localized along these walls.

Instead of bulk phases, droplets of liquid crystalline DNA may also be observed, usually in equilibrium with the isotropic phase (Fig. 4). These may be as large as a few tens of $\mu \mathrm{m}$ and therefore observable in optical microscopy.

A few points can be noticed:

- Droplets may be at equilibrium with a dilute ${ }^{24}$ (Fig. 4(c)) or a concentrated DNA isotropic phase ${ }^{29}$ (Fig. 4(a)), or with a solution containing other components such as 5\% PEG and single stranded DNA (Fig. 4(b)). ${ }^{28}$

- In all cases, the orientation of molecules at the interface of the droplets determines the shape of the droplet itself. In most cases, droplets are spherical, and the cholesteric layers (molecules) align parallel to the interface (Fig. 4). The 

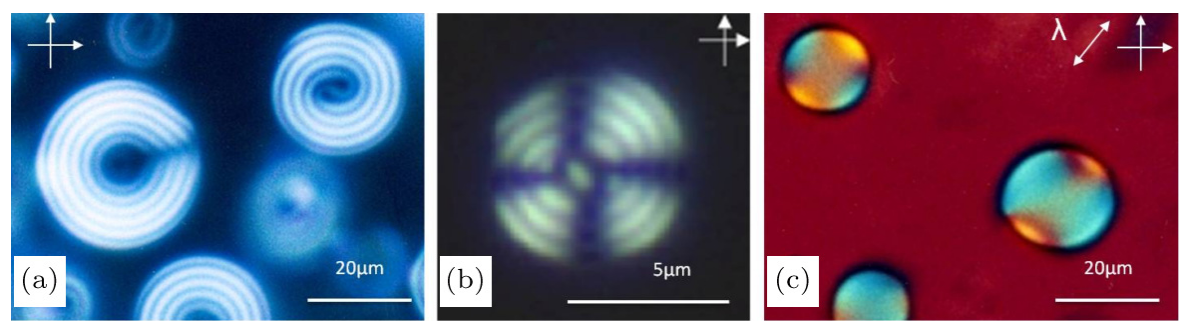

Fig. 4. Spherical cholesteric droplets of DNA in equilibrium with the isotropic phase. Samples were prepared with $50 \mathrm{~nm}$ DNA fragments in Tris buffer plus $\mathrm{NaCl}$ (a), and trivalent cations spermidine in (c). In (b, reprint from Ref. 28), samples are prepared with 294bp DNA fragments of defined sequence in $0.5 \mathrm{M} \mathrm{NaCl}$ and $5 \%$ PEG. Observations in polarizing microscopy; two $\lambda / 2$ plate have been added in (a) and a $\lambda$ plate in (c).

cholesteric axis is thus radial. Spherical droplets present an interesting structure showing double spiral patterns and under some favorable orientation their radius of disinclination. ${ }^{30,29,31}$

- The cholesteric pitch is independent of the dimensions of the droplet. It is small compared to the diameter in Fig. 4(a), Fig. 4(b), and large in Fig. 4(c).

\subsubsection{Structure of DNA in the bacteriophage capsid}

Many models of the organization of intracapsid DNA have been published but it is still an open question. In particular the path of the DNA chain remains unknown. The unique DNA chain is densely packed and organized locally in such a way that DNA segments form an hexagonal lattice revealed initially by X-ray diffraction ${ }^{32,33}$ and visualized later on by cryoTEM in several bacteriophages (see Refs. 34, 35 for example). The average inter-helix spacing $a_{H}$ is close to $2.6-2.7 \mathrm{~nm}$ in most bacteriophages. We want to stress here that cryoTEM conditions must be chosen carefully in order to visualize details of the DNA packing. These are well resolved at a particular value of under focus (see the picture of a T5 bacteriophage taken at an under focus value of $850 \mathrm{~nm}$ on Fig. 5 together with the simulated contrast transfer function of the microscope under this particular imaging condition). Average images of a large number of capsids taken under multiple orientations inside the thin film of vitrified water show concentric patterns better resolved close to the capsid and fuzzy in the center. ${ }^{36}$ They have often been misinterpreted as the presence of concentric layers of DNA when they only reveal that DNA segments are on average distributed at an equal distance from one and other (the distance $d$ between the stripes of the concentric patterns is linked to the inter helix distance $a_{H}$ by the relation $d=$ $\left.a_{H} \sqrt{3} / 2\right)$. These average images differ significantly from raw images of individual capsids showing patterns that differ from one capsid to the other.

In T5, each individual capsid appears as a mosaic of hexagonal domains ${ }^{37,38}$ separated by twist walls. ${ }^{39}$ The twist axis between hexagonal domains is radial and the distribution of the domains thus draws concentric patterns under favorable 


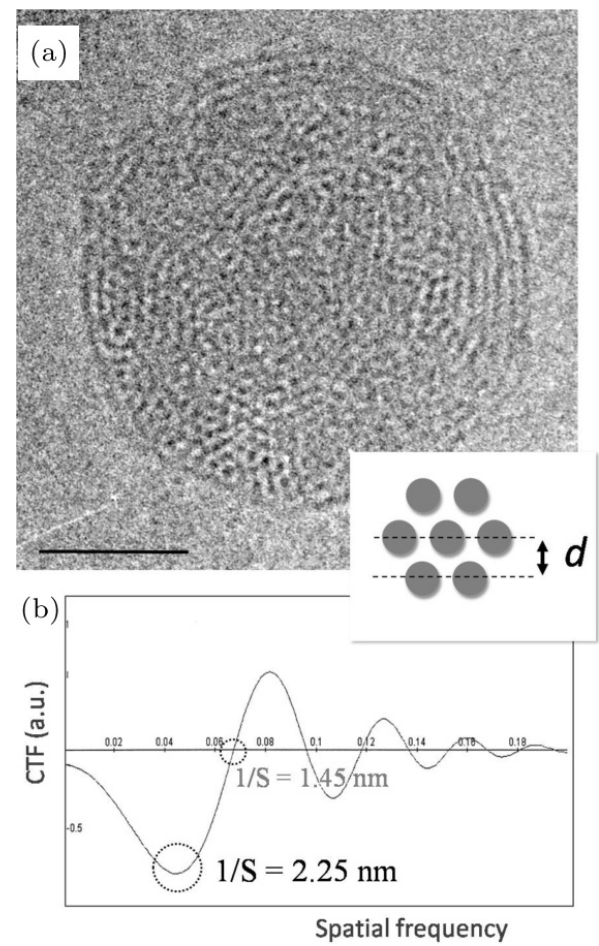

Fig. 5. (a) A cryoTEM image of bacteriophage T5 recorded at an underfocus value $(850 \mathrm{~nm})$ optimal to highlight the DNA lattice spacing in the capsid $d=a_{H} \sqrt{3} / 2$. (b) Simulation of the contrast transfert function (CTF) of the microscope at this underfocus (from http://ncmi.bcm.tmc.edu/homes/wen/ctf): the first maximum of the CTF is in the range of $1 / s=2.2-2.3 \mathrm{~nm}$, which is close to the lattice spacing of the DNA in the full capsid. Values close to $1.45 \mathrm{~nm}$ are vanished. Scale bar $=25 \mathrm{~nm}$.

orientations (underlined on Fig. 6(a)). Segments of the chain are aligned parallel to the capsid surface. This organization into domains separated by twist walls is very similar to the structure of bulk DNA described for the same range of helix spacings (Figs. 3(b) and 3(c)). A major difference is the nanometer dimension of the capsid that makes difficult the analysis of the structures. Hexagonal domains that may extend over tens of nanometers in Fig. 3(b) are reduced here to distances ranging from 5 to $16 \mathrm{~nm}$ (1-7 DNA segments aligned in parallel in each domain).

In vitro DNA ejection is a multi-step process initiated by the binding of the phage tail on its protein receptor. Once bound, a signal is transduced from the tail region in contact with the receptor to the head-to-tail connector, inducing the opening of a channel through the tail. As a result, DNA is released from the phage capsid into the external medium. Interestingly, it has been possible to image capsids trapped at intermediate states of DNA ejection and to reconstitute how the DNA chain, shorter and shorter as DNA release goes on, reorganizes and fully occupies the entire volume of the capsid (Fig. 7). The structure turns first 2D hexagonal, 

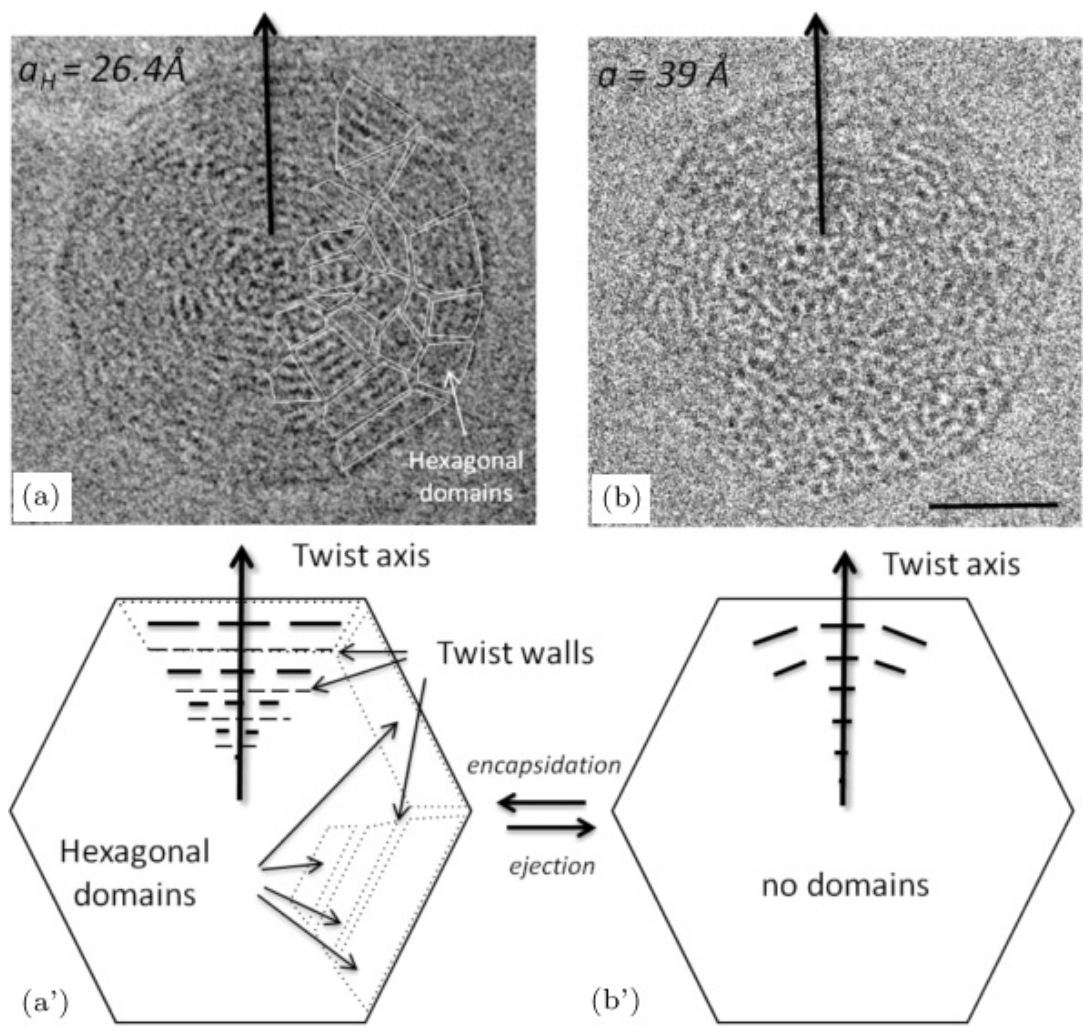

Fig. 6. T5 capsid either full (a) of after partial ejection of the chain (b): inter helix distances drop from 2.64 to $3.9 \mathrm{~nm}$ and the organization changes. The structure is still chiral, with a radial twist axis in both cases. DNA segments may be seen top view (dots) or in oblique/side views (lines) as explained in Fig. 3, and are represented respectively by dots and lines of increasing length in $\left(\mathrm{a}^{\prime}\right)$ and $\left(b^{\prime}\right)$. Bar $=25 \mathrm{~nm}$.

(Fig. 7(b)) and then cholesteric (Fig. 7(c)) and isotropic (Fig. 7(d)). The twist that was discrete between small domains in the full capsid becomes continuous in the less densely filled cholesteric capsid. In both cases, molecules are oriented parallel to the capsid (as in spherical DNA globules of Fig. 4(a)).

We see how these configurations are related to the structure of DNA droplets with noticeable differences anyway:

(i) DNA inside the capsid has reached concentrations higher than observed in spherulites.

(ii) DNA is confined and compressed in the capsid whereas the globule corresponds to an unconfined equilibrium state.

(iii) Capsids are significantly smaller than spherulites formed in solution.

(iv) Spherulites have been formed yet with short DNA fragments compared to the extremely long DNA chain of the bacteriophage. 


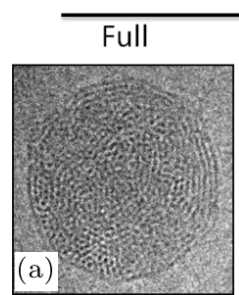

hexagonal

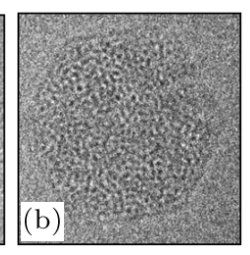

2D hexagonal

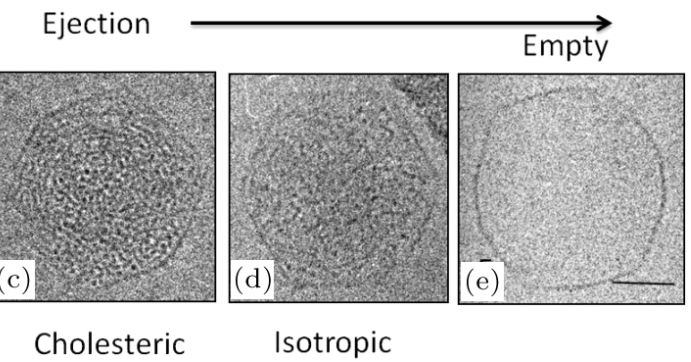

Fig. 7. Series of T5 capsids frozen at different steps of the DNA ejection. DNA always occupies the entire volume of the capsid. The DNA organization changes from 3D hexagonal to 2D hexagonal, cholesteric and isotropic. Bar $=25 \mathrm{~nm}$ (redrawn from Ref. 39).

(v) Additionally, DNA is packed inside an icosahedral capsid and the facetted capsid interferes with the DNA organization (by the introduction of additional defects $^{39}$ and possibly by protein structure and charges for example).

Nevertheless, these larger objects are useful to understand the rules that prevail in the organization of DNA and to reconsider them in the case of the bacteriophage.

\subsubsection{Single bacteriophage DNA ejection}

Different approaches have been used to analyze the kinetics parameters of the ejection steps. Bulk approaches (fluorometry and light scattering) give access to the kinetic parameters of the phage binding and ejection channel opening that constitute the rate limiting steps of the ejection. ${ }^{40,41}$ Compared to these first steps, DNA release occurs in a very short time (seconds compared to minutes) and the kinetics characterization relies on single phage assays using high speed time-lapse fluorescence microscopy. ${ }^{42,43,41,12}$ These experiments require the immobilization of the phage on a flow cell and the ejected DNA length is visualized by adding a fluorescent dye (Fig. 8).

Results reported in Chiaruttini et al. ${ }^{41}$ show that the full T5 genome is released in about $8 \mathrm{~s}$ (average time) with a distribution ranging from 3 to $20 \mathrm{~s}$. The velocity of DNA release has been calculated from single T5 ejection events, revealing regions where velocity drops down to zero, which means pauses (Fig. 9(b)).

Pauses will be described in the next section. The velocity averaged over all ejection events leads to a smoother profile with a non-monotonic behavior as a function of the ejected length (Fig. 9(a)). The maximum speed and the location where it is reached depend on the ionic conditions: the more repulsive the interactions (less screening), the higher is this speed with a location of the maximum at relatively low DNA content. In contrast, the maximum speed is reached at higher DNA content when interactions are screened (less repulsive). For T5 in a monovalent buffer (100 mM NaCl, $1 \mathrm{mM} \mathrm{MgSO}_{4}, 1 \mathrm{mM} \mathrm{CaCl} 2,10 \mathrm{mM}$ Tris, $\mathrm{pH} 7.2$ ), a maximum speed between 60 and $120 \mathrm{kbp} / \mathrm{s}$ is reached for $35 \%$ ejected DNA. In divalent buffer 

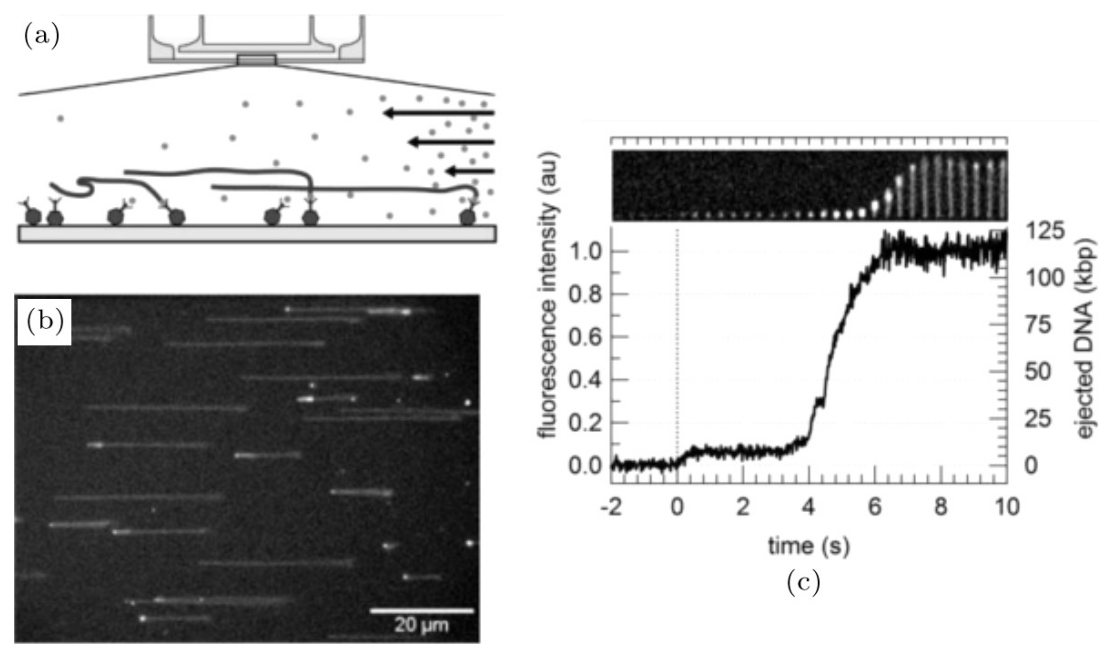

(c)

Fig. 8. Schematic representation of the microscopy flow chamber and overview of representative fluorescent images. (a) Phage T5 $\left(6 \times 10^{6}\right.$ particles) were allowed to adsorb on the bottom of a flow chamber thermostated at $23^{\circ} \mathrm{C}$. FhuA $(0.8 \mu \mathrm{g})$ and YOPRO-1 $(0.1 \mu \mathrm{M})$ were injected into one of the compartments connecting the central channel. The DNA was stretched by applying a hydrodynamic flow of buffer by means of a peristaltic pump connecting the two compartments. (b) Overview of one of the frames 2 min after injection of YOPRO-1 and FhuA and application of the flow buffer. The frames show that discrete lengths of T5 DNA were ejected (from Ref. 43). (c) Time series of a single phage beginning the ejection of its DNA at $t=0$. The length of the ejected DNA is computed by measuring the total fluorescence intensity integrated along the DNA molecule. (from Ref. 41)

$\left(10 \mathrm{mM} \mathrm{MgSO}_{4}, 10 \mathrm{mM}\right.$ Tris, $\left.\mathrm{pH} 7.6\right)$ the maximum speed decreases to $10 \mathrm{kbp} / \mathrm{s}$ and is shifted to $25 \%$ ejected $\mathrm{DNA}^{44}$ (Fig. 10(a)). For Lambda in Na buffer, ${ }^{42}$ a maximum value of $50 \mathrm{kbp} / \mathrm{s}$ is reached, whereas the maximum speed is lowered to $17 \mathrm{kbp} / \mathrm{s}$ in presence of $\mathrm{Mg}^{2+}$ (Fig. 10(b)). The total ejection time is also sensitive to buffer. It increases from $1.5 \mathrm{~s}$ in $\mathrm{Na}$ buffer to $8-11 \mathrm{~s}$ in $\mathrm{Mg}^{2+}$ buffer for Lambda and from $8 \mathrm{~s}$ in $\mathrm{Na}$ buffer to $50 \mathrm{~s}$ in $\mathrm{Mg}^{2+}$ buffer for $\mathrm{T} 5$.

Therefore, the velocity of exiting DNA increases up to a given value and then slows down, in $\mathrm{T} 5^{41,44}$ and in Lambda. ${ }^{42}$

\subsubsection{Pauses and intermediate states of DNA ejection}

- Observations by fluorescence microscopy. Pauses during T5 DNA release were reported in two studies. ${ }^{43,41}$ Both sets of data agree well except a distribution of partial lengths located close to half genome reported in the first case ${ }^{43}$ that was not observed in the second one. ${ }^{41}$ The main difference between both studies comes from the method used to estimate the ejected DNA amount. It was estimated by the direct measurement of the linear length of the ejected DNA stretched in a flow (Fig. $8(\mathrm{~b}))^{43}$ or by measuring the total fluorescence intensity integrated along the DNA molecule (Fig. 11). ${ }^{41}$ This last approach gives a better precision of 
(a)

$$
\text { ejected DNA (kbp) }
$$

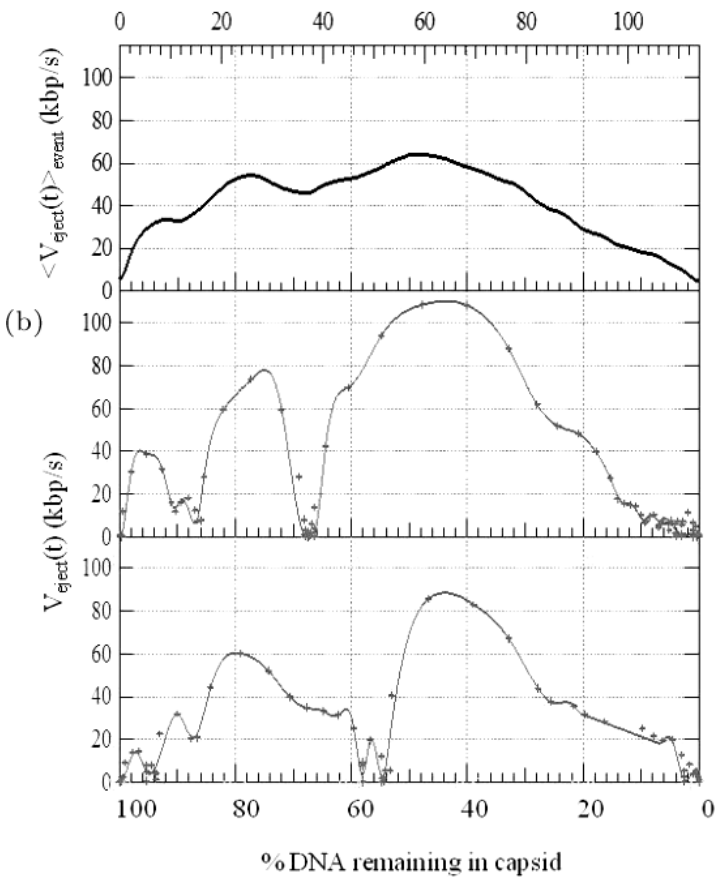

Fig. 9. (a) Averaged DNA release velocity as a function of ejected DNA length for T5. (b) Two individual velocity plots calculated from a single T5 ejection (from Ref. 41).

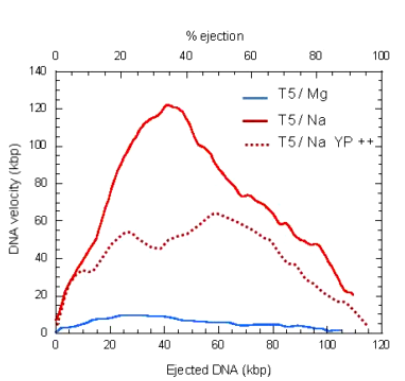

(a)

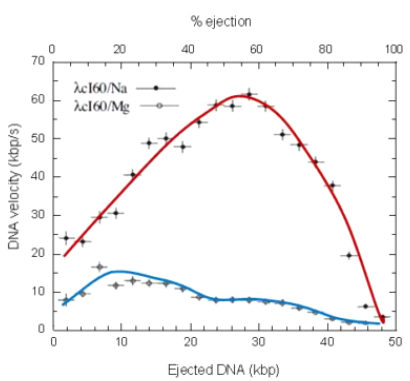

(b)

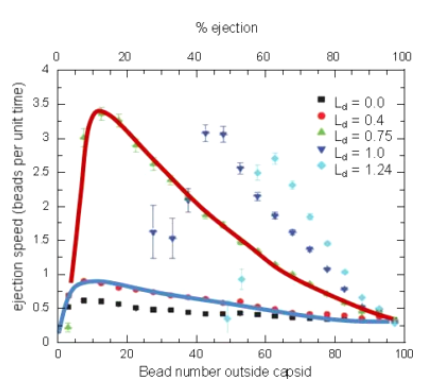

(c)

Fig. 10. (Color online) Comparison of ejection speeds in experiments (T5 in a, Lambda in b) and in simulations (c) as a function of the amount of DNA (or beads) within the capsids and for various ionic environments. Red curves were recorded under more repulsive interactions $\left(\mathrm{Na}^{+}\right.$ cations) compared to blue ones $\left(\mathrm{Mg}^{2+}\right.$ cations). In (a), the speed is increased when less fluorescent dye is added to the sample (dashed red curve). ${ }^{44}$ In (b), the variation of Lambda ejection velocity is caused by the difference in buffer conditions (from Ref. 42). In (c), the curves (from black to light blue symbols) consider increasing values of the ionic strength (or equivalently of the Debye screening length) (from Ref. 45). 


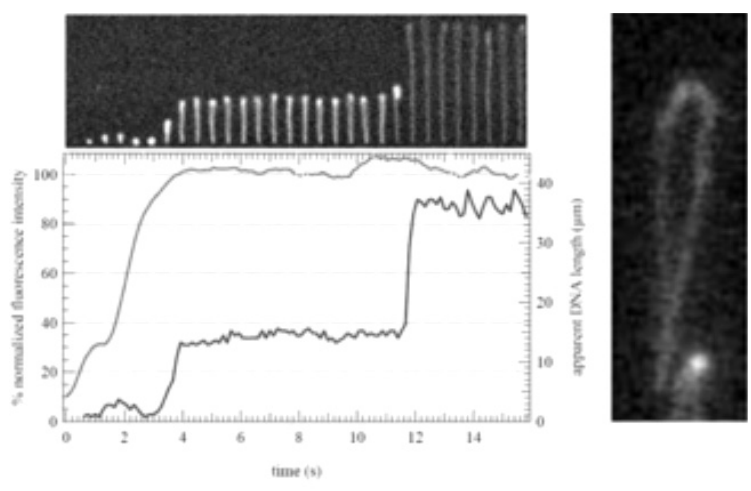

(a)
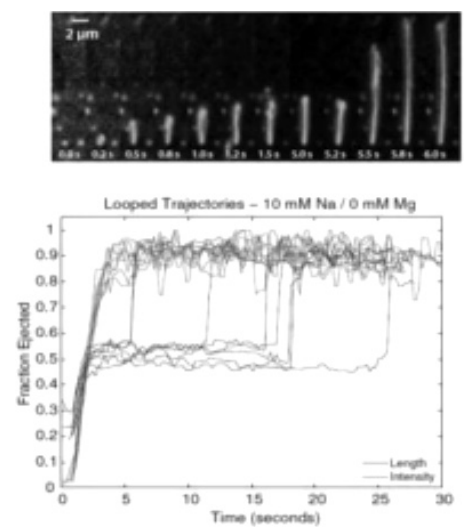

(b)

Fig. 11. (a) Time series images of one phage T5 releasing its DNA. About $12 \mathrm{~s}$ after DNA release initiation, the bright short DNA molecule becomes longer and less intense. The graph below shows that although the DNA length increases suddenly (lower curve) the fluorescence intensity remains constant (upper curve). The interpretation is that while DNA is ejected, it is attached by both ends, forming a loop. DNA loop is visible in time series taken at high magnification. ${ }^{41}$ (b) Looped ejections were also observed for phages Lambda. ${ }^{46}$

the ejected amount than direct length measurements. The analysis of the partial lengths close to $50 \%$ (initially attributed to pauses in Ref. 43) shows that they are related to the formation of a loop by the ejected DNA molecule. In time series taken at high magnification, a loop formed by DNA attached by both ends is clearly visible (Fig. 11(a)). Looped ejections were also observed for phage Lambda and they exhibit the same dynamics as unlooped ejections (Fig. 11(b)). ${ }^{46}$

In T5, 90\% of the phages eject their DNA by stochastic bursts of partial ejection separated by transient pauses (Fig. 12(a)). Pauses have also been observed for His1, an archeal virus ${ }^{47}$ but have not been detected in lambda phages. The reasons for that remain unknown. A unique feature of $\mathrm{T} 5$ is the existence of genetically defined single stranded interruptions (nicks) in one of the DNA strands. ${ }^{48-50}$ The influence of the nicks on DNA release was excluded because a T5 nickless mutant was shown to eject its DNA with the same stepwise behavior than the wild-type phage (Fig. 12(b)).

If we hypothesize that pauses are related to local DNA rearrangements within the T5 head, we can expect other phages with a similar packing density to also display transient ejection stops when observed with the same accuracy. The difference between T5 and Lambda could be related to a difference in experimental setup: an EMCCD detection was used for T5 analysis allowing higher acquisition rates (62 fps) compared to Lambda (4 fps). If for some unknown reasons, Lambda arrests are shorter than T5 ones, they could not have been detected at this lower acquisition rate. Pause duration could also be sensitive to different parameters (buffer composition, nature and concentration of DNA fluorescent 


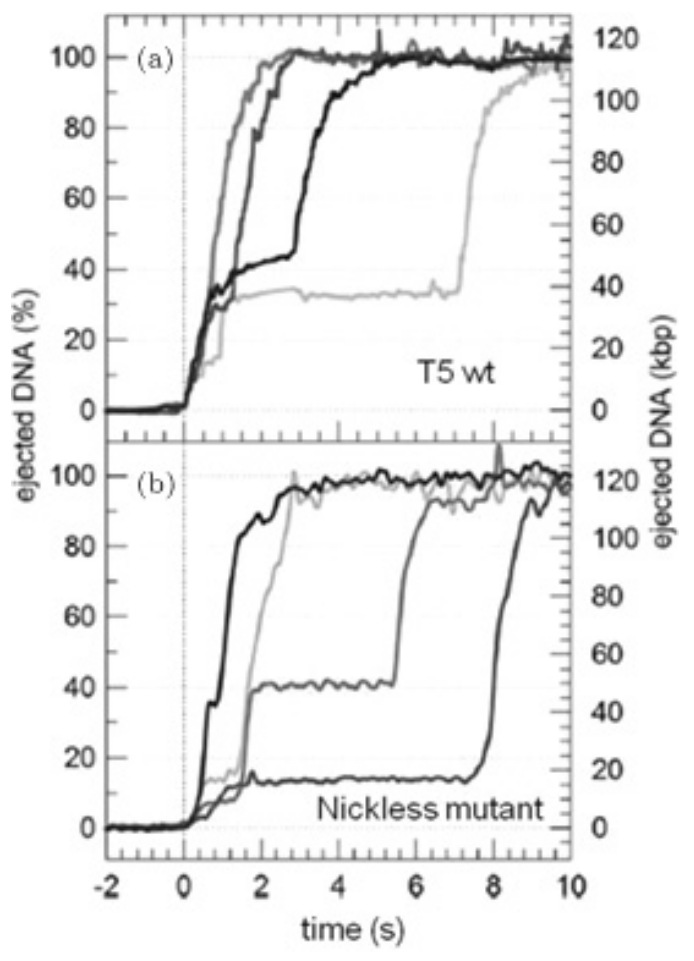

Fig. 12. Typical traces of DNA release kinetics measured on single T5 phage. Similar curves are obtained on wild-type phage (top curves) and nickless mutant (bottom curves) (from Ref. 41).

dye, etc.). For example, in T5 ejection experiments, a decrease of the dye concentration results in the observation of shortened pauses or slow-down points with a non-zero speed ${ }^{44}$ (Fig. 10(a), dashed red curve). As a consequence, depending on experimental conditions, very short pauses could not be detected. By exploring a larger range of conditions, we may found ionic conditions where T5 does not pauses and others where Lambda does.

- Comparison of pauses observed in fluorescence microscopy and in cryoTEM. In fluorescence microscopy, the full T5 genome is released in about $10 \mathrm{~s}$ and recorded pauses occur in this time range. The analysis of 62 individual ejections indicates that pauses are located around three different regions: a sharp peak at $6 \%$ and two broader ones at $12 \%$ and $40 \%$ ejected DNA (Fig. 13(a)). The precision in length determination (few $\mathrm{kbp}$ ) is not sufficient to determine whether pauses occur during the last few percent of DNA length. In cryoTEM experiments, samples are frozen before the ejection is completed for all phages. Many capsids contain intermediate amounts of DNA while some are already empty and others still full. Statistically, those partially filled capsids can be considered as transiently stopped at a pause because measured DNA lengths are not equally distributed over the genome length. Pauses revealed by cryoTEM depend on a 


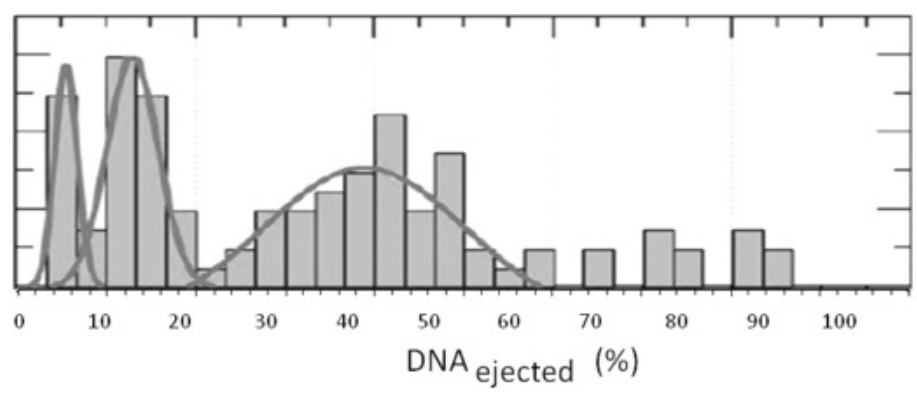

(a)

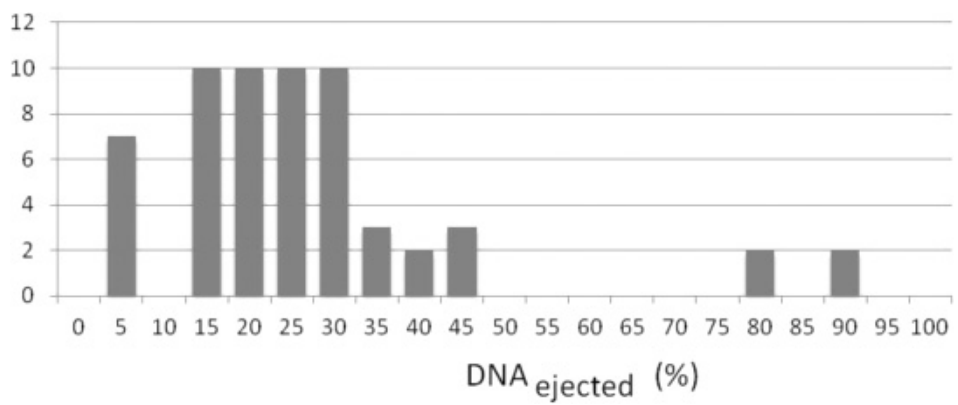

(b)

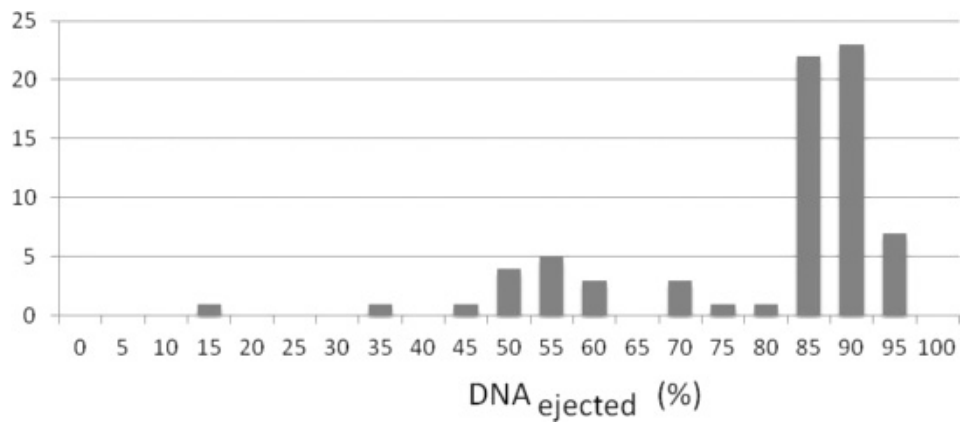

(c)

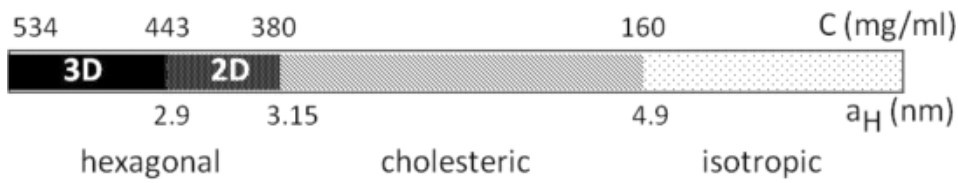

(d)

Fig. 13. Histograms of the pauses location detected along the genome of T5. (a) Pauses detected by fluorescence (redrawn from Ref. 41). (b, c) Pauses detected by cryoTEM when phages are adsorbed onto a carbon surface (b) or when phages are free in the bulk (c). (d) The multiple phases formed by short DNA fragments are indicated using the same DNA concentration scale, ranging from $530 \mathrm{mg} / \mathrm{ml}$ (full capsid, left) to the empty one (right). Ejection protocols are very similar in (a) and (b): receptors were prefixed onto the phages at $4^{\circ} \mathrm{C}$ (no permissive ejection) and deposited on the surface (plastic of the flow cell for (a) and carbon film in (b)) before transfer to $37^{\circ} \mathrm{C}$. In (c), phages allowed to eject DNA in the bulk are secondarily trapped in a thin film of water for freezing and imaging. See experimental details in Ref. 39. 
combination between the frequency and the duration of each pause. For a given frequency, we therefore detect preferentially long pauses compared to short ones. Pauses are mostly recorded at high content (5-45\% ejected DNA) (Fig. 13(b)), when phages are adsorbed on a carbon surface and at lower DNA content (two peaks around 55 and $90 \%$ ) when phages are free in the bulk ${ }^{39}$ (see experimental details in the legend of Fig. 13(c)). Pauses are also detected with a much lower frequency in other positions in both conditions.

Interestingly, upon adsorption on a surface (plastic of the flow chamber in fluorescence experiments and carbon film in cryoTEM experiments), pauses happen when less than half of the genome is ejected. The fact that pauses happen when the second half of the ejection takes place in non-adsorbed phages let us suspect that interactions of the capsid with the surface has a significant incidence on the occurrence of these pauses. The comparison of the three sets of data (Fig. 13) indicates that the influence of the surface is comparable in fluorescence and cryoTEM experiments. Minor differences in the pause distribution between the two experiments could be related (1) to the precision of the length measurements ( $2 \mathrm{kbp}$ in fluorescence microscopy versus $5-10 \mathrm{kbp}$ in cryoTEM) as well as to different experimental conditions such as the presence of DNase in cryoTEM and of course not in fluorescence microscopy and (2) to the absence of fluorescent dye in cryoTEM. Despite all these differences, the good agreement between both sets of data confirms that pauses are not artefacts induced by experimental settings.

- Arrested states of DNA ejection induced by osmolites or condensing agents. In the case of Lambda and SPP1, ejection experiments in the presence of PEG show that the amount of ejected DNA monotonically decreases with the increase of the applied pressure. ${ }^{51,17} \mathrm{~T} 5$ shows a very different behaviour. When the ejection is triggered at low and intermediate pressure, the phage population does not behave homogeneously. Different sub-populations corresponding to discrete amounts of ejected DNA coexist, some of which even show a complete ejection. ${ }^{52}$ In addition to the empty phages, two major sub-populations have been reported (Fig. 14(a)), around 50-55 and 90\% ejected DNA (with an additional very minor population around $70 \%$, not shown), independent of the applied pressure. For pressures above 7 atm, these sub-populations disappear and T5 behaves as Lambda and SPP1. The same states are detected when the ejection is triggered in the absence of pressure but in the presence of condensing amounts of the tetravalent cation spermine (Fig. 14(b)). Two additional sub-populations are also found: (1) the population of empty phages found by cryoTEM (Leforestier, unpublished results) and (2) the population of phages that eject a small amount of DNA to reach the equilibrium state in presence of condensing spermine $(80-100 \mathrm{kbp}) .{ }^{53}$ We may hypothesize that the two bands (around $45-50 \mathrm{kbp}$ and $11 \mathrm{kbp}$ ) are not exactly identical to those observed in the presence of PEG (Fig. 14(a)) because the DNA organization differs in the two experimental conditions. ${ }^{54}$ Interestingly, these intermediate states roughly correspond to the location of the pauses detected by cryoTEM 


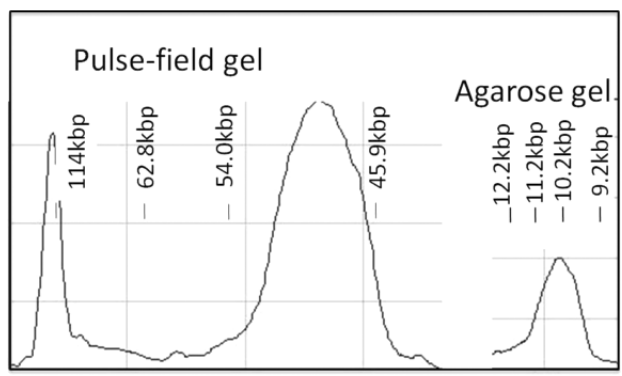

(a)

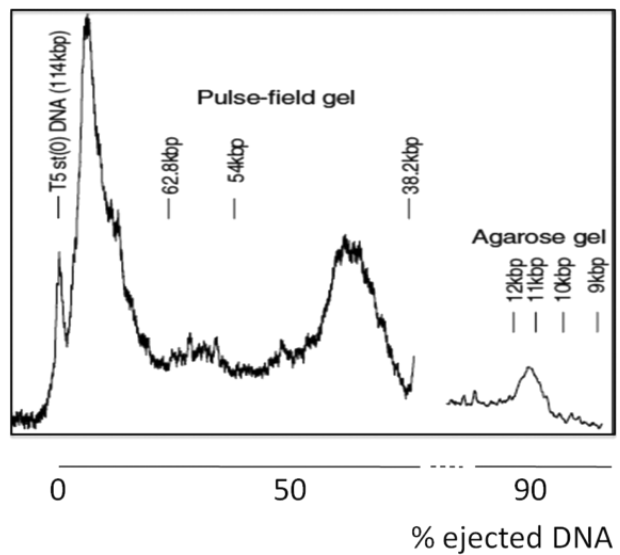

(b)

Fig. 14. Electrophoresis profiles of the DNA length distribution remaining in the capsid after the ejection has been triggered in the presence of an external pressure obtained with PEG (a, $P_{\mathrm{PEG}}=4.99 \mathrm{~atm}$; redrawn from Ref. 52) and in the presence of a condensing amount of spermine (b, $C_{\text {spermine }}=1 \mathrm{mM}$; redrawn from Ref. 53 ). In both cases two populations are detected roughly centred around 45-50 kbp (Pulse-field electrophoresis) and $11 \mathrm{kbp}$ (Agarose gel) respectively. We do not focus here on the bands at $114 \mathrm{kbp}$ (full phages in a and b) and around $100 \mathrm{kbp}$ in (a particular situation discussed elsewhere, corresponding to the equilibrium state in the presence of condensing spermine in b). Experiments were realized in the presence of DNase to digest the ejected fraction of DNA.

when the phages eject in bulk solution (see histogram on Fig. 13(c)). The pauses in $\mathrm{T} 5$ therefore correspond to robust transient states that can be trapped by arresting the ejection in the presence of any agent that can interfere with the DNA organization.

\subsection{Theoretical studies}

Diverse models have been proposed to describe the DNA encapsidation and ejection processes. A first group of theoretical studies based on statistical mechanics uses a continuum representation of DNA. ${ }^{55-57}$ By including electrostatic and elastic contributions to the energy, these methods reproduce the monotonical variation of 
the packing force as a function of the amount of encapsidated DNA observed in packing experiments with different phages (phi 29, T4, Lambda). ${ }^{9,8,10,58}$ In these studies, the ejection is considered as a quasi-static mechanism and the force driving ejection is supposed to be equal to the equilibrium value of the force acting against DNA encapsidation. These approaches show that the ion dependence of the ejection dynamics is a direct consequence of the modification of DNA interactions. ${ }^{46}$ However they fail to reproduce the experimental non-monotonical variation of the ejection speed (Figs. 10(a) and 10(b)): the intra-capsid pressure reaches its maximum value for a fully packed capsid and decreases monotonically as ejection proceeds, in contrast with the measured speed that is maximum for an intermediate DNA fraction. Grayson et al. ${ }^{42}$ explained this variation of the speed by introducing a friction coefficient $\phi=F / v$ dependent on the amount of DNA inside the capsid, and coming from hydrodynamic drag within the phage head. In this description, it remains to understand the exact physical origin of the friction coefficient and to predict its magnitude. Discrepancies between these predictions and experiments reveal the importance to go beyond the quasi-static description and to consider ejection as a non-equilibrium process that competes with the relaxation processes of the DNA organization inside the capsid.

An alternative point of view comes from the simulations performed within the framework of coarse-grain representations of DNA by means of various computational techniques (Molecular Dynamics, Stochastic Rotational Dynamics and Langevin Dynamics) (see references in the introduction from Refs. 59 and 45). These theoretical approaches predict the time scale of the packaging and ejection processes in relation with DNA rearrangement within the capsid. The force resisting packing is different from the ejection force due to dynamical effects and reflects the importance of non-equilibrium (or dynamics) versus equilibrium (or thermodynamic) effects on the packaging/ejection problem. ${ }^{45}$ In contrast with DNA-DNA electrostatic repulsion, packing and ejection forces appear to vary non-monotonically with the encapsidated fraction due to a non-trivial interplay between entropic and electrostatic forces. ${ }^{45,60}$ The associated ejection speed is slow at maximum packing, increases to a maximum value and finally decreases as ejection proceeds: the system behaves as if DNA might initially bring itself to a configuration favorable for ejection before the process becomes fast and efficient. The maximum value of the ejection speed and its position strongly depend on ionic conditions (Fig. 10(c)) because getting to the favorable configuration is more difficult when electrostatic interactions are involved. Indeed, the fluctuations needed to change the polymer conformation are hampered by the extra repulsion among the polymer beads. Different authors have investigated the influence of the bending rigidity on the ejection process. The ratio between the polymer persistence length and the size of the capsid appears to be an important parameter for the ejection process. ${ }^{60,61}$ These models also predict the existence of pauses during DNA ejection, randomly distributed along the genome. ${ }^{62,60,61}$ Their origin is associated to temporary blockages due to the DNA configuration at the exit of the capsid. Pauses have been detected in packaging experiments of Lambda ${ }^{63}$ 
especially at higher forces and also in simulations. As suggested by Ali et al. ${ }^{45}$ pauses reported in encapsidation and ejection may be of similar nature and their duration would be a function of the driving force for the ejection.

In contrast with the first approaches that have no predicting power regarding the genome structure and assume a spool organization within the capsid, simulations do not rely on an a priori structural model and lead to structure predictions. By including stretching and bending terms, DNA-DNA interactions and a DNA-capsid volume exclusion terms in a coarse-grain model, Petrov et al. ${ }^{64}$ showed that in a isomeric capsid without core, genome is packaged in successive layers to minimize the bending stress, leading to a concentric arrangement. With the increase of the internal pressure as more and more DNA is encapsidated, the internal motion of DNA diminishes and the coaxial spool motif becomes distorted. This leads to considerable variation between different packaging trajectories. In another study, ${ }^{59}$ these authors show that there is no substantial difference in the internal energy between co-axially or concentrically spooled and folded-toroidal conformations. This means that different conformations of DNA could coexist within a phage population.

Recent molecular dynamics simulations, based on the stochastic rotation algorithm, ${ }^{45}$ investigate the effect of ions on flexible and semi-flexible polymers, packed in a spherical capsid. The semi-flexible polymer arranges in loops (with the sections that entered the capsid earlier arranged quasi-randomly in the capsid centre) while the flexible polymer is in a random configuration. These results illustrate the impact of chain rigidity on DNA organization. Ionic effects, although having a massive effect on the ejection dynamics, do not appreciably change the DNA configuration which is crucially determined by the rigidity and other local potential fields (such as the almost parallel alignment). ${ }^{45}$

A recent study also analyzes the stochasticity of the ejection kinetics. ${ }^{61}$ The DNA is encapsidated and ejected through a pore (the motor protein complex) located at one apex of the capsid. This pore is described as a hollow cylinder with an internal diameter close to the DNA size. The orientation of the DNA close to the pore dictates the instantaneous ejection speed; an important angle between the chain and the pore axis induces slowing down and pauses. Highly stochastic variations of the ejection kinetics are thus observed, reflecting the non-equilibrium conformations of initially packed DNA inside the full capsid. The distance of the DNA conformation from equilibrium state appears to vary with the chain flexibility and with the motor forces that have been applied during DNA packaging. A low packaging force leads to a slower encapsidation process, a better ordering of the packed chain and a final DNA configuration at equilibrium or close to equilibrium.

\section{Discussion and Conclusions}

Although all full bacteriophage capsids of a given species contain an identical length of DNA trapped in a defined volume, with a precise interdistance between hexagonally ordered DNA strands, each capsid is unique. There is not a unique 
path of the chain inside the capsid. This multitude of paths does not preclude the existence of rules in the DNA packing process, mostly imposed by the properties of the chain (structure, sequence, charge), and its environment (volume confinement, nature and concentration of counter ions and added salts). These rules are far from being understood because certain relevant parameters have not been considered in theoretical descriptions. Indeed, all models consider an idealized Worm Like Chain Model (WLC) for the DNA chain, with an average persistence length of about $150 \mathrm{bp}(50 \mathrm{~nm})$. This view is incorrect. Cloutier and Widom ${ }^{65,66}$ showed that the DNA molecule is inherently very flexible, and that sharp bends occur spontaneously, allowing sequences as short as $94 \mathrm{bp}$ to form mini circles. As a consequence, almost all numerical simulations predict an heterogeneous distribution of the DNA inside the capsid, generally with an accumulation at the periphery where the molecule spools into concentric (or coaxial) layers. So far, there is no model to account for the homogeneous DNA distribution observed experimentally at all steps of the ejection process. This may be due to an overestimation of the elastic energy, and also to the fact that they do not take into consideration important parameters dealing with the secondary structure of the molecule, namely the possibility that the chain forms kinks or adopts transient non-canonical configurations (denaturation or flipping out of the base pairs) reported in experiments and simulations of mini circles submitted to intense stress, ${ }^{67-69}$ review in. ${ }^{70}$ They do not consider either parameters specific to extreme conditions of concentration and confinement encountered by the DNA chain inside the capsid such as (1) correlations between the position of helices when they are forced to come very close together as evidenced experimentally by X-ray ${ }^{7}$ and cryoTEM ${ }^{71}$ and predicted theoretically, ${ }^{72}(2)$ geometric frustrations that arise from the competition between a dense hexagonal packing and the impeded spontaneous twist originating from the helicity of the molecule that imposes strong constraints to the geometric configurations of the dense phase ${ }^{73,25}$ and also (3) the possible variation of the DNA helical pitch when correlated helices are very densely packed and/or strongly curved. ${ }^{7,71}$ In samples prepared with short DNA fragments, the helical pitch $(0.34 \mathrm{~nm}$ in solution) is significantly decreased at the concentration found in the full capsid. ${ }^{7}$ There is an open field now to explore how DNA packs inside capsids of different sizes. Things are moving, with the introduction of more flexibility for the chain in MD simulations of genome packaging and ejection with no a priori information on the initial structure. ${ }^{45}$ Petrov et al. ${ }^{64}$ also report a transition from an isotropic to a liquid crystalline (nematic) phase with the increase of DNA concentration during encapsidation. But significant differences with experiments can be pointed out, especially the fact that only one transition is predicted, at high DNA filling ( $80 \%$ full capsid) instead of three observed experimentally, at about 30 and 70 and $85 \% .{ }^{39}$

Concerning the kinetics of DNA ejection, the non-monotonous variation of the velocity observed under non-condensing conditions is consistent with recent theoretical models that include a continuous reorganization of DNA inside the capsid during ejection (Fig. 10). Pauses have been predicted, ${ }^{62,61}$ arising from a temporary 
blockage of the chain at the exit. These blockages would result from an inadequacy between the ejection force and the ease of the chain to be mobile and to reorganize inside the capsid to be in a favorable conformation to go out. These pauses are predicted to be evenly distributed along the genome ${ }^{45}$ in contradiction with observations in T5. We cannot exclude that the pauses observed experimentally are the longest ones and that shorter ones were not detected. Following Ali et al., ${ }^{45}$ all experimental parameters (ionic conditions, presence or absence of dye and DNase, adsorbed/unadsorbed phages etc.) with the ability to modify the energy of the system could have an influence on the ejection velocity and the observed pauses distribution. It is not straightforward to predict the exact effect of each parameter that vary for each set of data and we do not think it is relevant to compare predicted and observed distribution with a high accuracy. But we want to point out that despite the diversity of experimental conditions, pauses are always detected; their presence is thus a robust phenomenon.

However, how can we explain that pauses were not detected in vitro for Lambda? In a more general way, what is the origin of the differences between bacteriophage species? Despite the fact that the length of the genome and the diameter of the capsids may vary considerably $(48.5 \mathrm{kbp}$ DNA in a Lambda capsid about $50 \mathrm{~nm}$ in diameter compared to $113.9 \mathrm{kbp}$ T5 genome kept in a capsid $80 \mathrm{~nm}$ in diameter for example), the inter-helix spacing stays remarkably constant $(2.6-2.7 \mathrm{~nm})$ which implies a significant variation of the bending contribution. Other parameters can also distinguish the different phages: shape and charge of the capsid, length of the tail, force and speed of DNA encapsidation, etc. All these parameters can have an influence on the exact DNA organization within the phage, the energy balance and the dynamics associated with the ejection. ${ }^{74-76}$ For example if the encapsidation force is larger for $\mathrm{T} 5$ than for Lambda, we may expect more out of equilibrium states in T5 that would explain the observed pauses during ejection. As already discussed, all these parameters will result in a modification of the pause duration. Concerning the experimental detection of the pauses, it is most likely that conditions have to be carefully adjusted for each phage species. CryoTEM approaches that preclude any dye addition seem to be the more favorable way to look for them.

Whatever the details of DNA structures, each full capsid stores a given energy. If DNA encapsidation occurs too fast, the energy may be larger than the energy corresponding to the equilibrium state. ${ }^{61}$ These authors point out the non-ergodic nature of ejection kinetics. The stochasticity arising from the initial state of encapsidated DNA has consequences on individual ejection, as illustrated by the multiplicity of ejection trajectories and velocity profiles. If the energy stored in the full capsid is higher than expected for the equilibrium state (defined by the concentration and confinement), then the length of the ejected DNA may be longer than predicted. Experimental data that remained unexplained yet for ejection of T5 under applied external pressure or in the presence of a condensing agent like spermine $(4+)$ may be reconsidered. The ejected DNA lengths show discrete values, all larger than expected for an equilibrium of pressures inside and outside of the capsids. These 
would correspond to phages for which an additional energy was stored in the capsid. We measured DNA fragments of 11 and $50 \mathrm{kbp}$ trapped in the capsids (Fig. 14) which are also the locations of the pauses. Why does ejection stopped at such precise steps? Our interpretation is that there are several local energy minima where ejection of a given phage can be stalled or not, depending on its initial energy. We may wonder whether these minima correspond to special geometric configurations. It seems difficult to get estimates of the initial energy stored in such capsids, considering that many experimental parameters are not determined with enough accuracy for each individual phage.

In conclusion, we have investigated the relation between the ejection of bacteriophage DNA and its organization within the capsid by compiling and analyzing recent experimental and theoretical results. This work highlights the role of DNA organisation inside the bacteriophage capsid on the stochastic and out-ofequilibrium nature of the ejection process. Although the DNA transfer in vivo is much more complex than in vitro analyzed here and relies on a variety of mechanisms involving the host metabolism, we show that DNA organization within phage capsids could constitute a new molecular mechanism at the origin of phage adaptability. Adaptation to environmental stochasticity is a recurrent situation commonly faced by living organisms. It has been shown that the population heterogeneity can improve the fitness. ${ }^{77,78}$ For phage infection, several mechanisms have already been identified as a source of stochasticity: (i) the host expression of phage receptors that imposes the phage binding probability ${ }^{79}$ : (ii) the variation of the lysis time related to holing protein expression and diffusion ${ }^{80,81}$; (iii) the decision at a subcellular level between lysis and lysogeny. ${ }^{82,83}$ Concerning the phages, DNA organisation inside the capsid is a new source of stochasticity in addition to the variability in adsorption rate (possibly due to variable folding of tail fiber proteins ${ }^{81}$ ). Although initially homogeneous in terms of genotype, the phage population can generate different sub-populations with radically different phenotypes, exemplified here by the different amounts of ejected DNA and the stochasticity of the ejection kinetics which could play a role in the adaptation to environmental and host variability.

\section{Acknowledgments}

This work was supported by ANR-12-BSV5-0023-01. We thank Nicolas Chiaruttini for fruitful discussions.

\section{References}

1. C. M. Knobler and W. M. Gelbart, Annu. Rev. Phys. Chem. 60, 367-383 (2009).

2. W. M. Gelbart and C. M. Knobler, Physics Today 61, 42-47 (2008).

3. D. E. Smith, Current Opinion In Virology 1, 134-141 (2011).

4. A. S. Petrov and S. C. Harvey, Biophysical Journal 95, 497-502 (2008).

5. L. W. Black and V. B. Rao, Adv. Virus Res. 82, 119-153 (2012).

6. A. S. Petrov, S. S. Douglas and S. C. Harvey, J. Phys. Cond. Matt. 25, 115101 (2013).

7. D. Durand, J. Doucet and F. Livolant, Journal de Physique 2, 1769-1783 (1992). 
8. J. Kindt, S. Tzlil, A. Ben-Shaul and W. M. Gelbart, Proc. Natl. Acad. Sci. USA 98, 13671-13674 (2001).

9. D. E. Smith, S. J. Tans, S. B. Smith, S. Grimes, D. L. Anderson and C. Bustamante, Nature 413, 748-752 (2001).

10. P. K. Purohit, M. M. Inamdar, P. D. Grayson, T. M. Squires, J. Kondev and R. Phillips, Biophysical Journal 88, 851-866 (2005).

11. V. Zarybnicky, Journal of Theoretical Biology 22, 33-42 (1969).

12. D. Van Valen, D. Wu, Y. J. Chen, H. Tuson, P. Wiggins and R. Phillips, Current Biology 22, 1339-1343 (2012).

13. V. Zarybnicky and A. Zarybnicka, Virology 54, 318-329 (1973).

14. P. Boulanger, M. leMaire, M. Bonhivers, S. Dubois, M. Desmadril and L. Letellier, Biochemistry 35, 14216-14224 (1996).

15. L. Randall-Hazelbauer and M. Schwartz, Journal of bacteriology 116, 1436-1446 (1973).

16. M. Roa and D. Scandella, Virology 72, 182-194 (1976).

17. C. Sao-Jose, M. de Frutos, E. Raspaud, M. A. Santos and P. Tavares, Journal of Molecular Biology 374, 346-355 (2007).

18. C. Robinson, Tetrahedron 13, 219-234 (1961).

19. V. Luzzati and A. Nicolaieff, Journal of Molecular Biology 7, 142-63 (1963).

20. L. S. Lerman, Cold Spring Harbor Symposia on Quantitative Biology 38, 59-73 (1974).

21. F. Livolant and A. Leforestier, Progress In Polymer Science 21, 1115-1164 (1996).

22. G. S. Baldwin, N. J. Brooks, R. E. Robson, A. Wynveen, A. Goldar, S. Leikin, J. M. Seddon and A. A. Kornyshev, J. Phys. Chem. B 112, 1060-1064 (2008).

23. G. Zanchetta, F. Giavazzi, M. Nakata, M. Buscaglia, R. Cerbino, N. A. Clark and T. Bellini, Proc. Natl. Acad. Sci. USA 107, 17497-17502 (2010).

24. J. Pelta, D. Durand, J. Doucet and F. Livolant, Biophysical Journal 71, 48-63 (1996).

25. A. Leforestier, A. Bertin, J. Dubochet, K. Richter, N. S. Blanc and F. Livolant, Comptes Rendus Chimie 11, 229-244 (2008).

26. J. W. Goodby, Current Opinion In Colloid and Interface Science 7, 326-332 (2002).

27. A. Leforestier and F. Livolant, Biophysical Journal 65, 56-72 (1993).

28. A. Goldar, H. Thomson and J. M. Seddon, J. Phys.: Cond. Matt. 20, 035102 (2008).

29. Y. Bouligand and F. Livolant, Journal de Physique 45, 1899-1923 (1984).

30. C. Robinson, J. C. Ward and R. B. Beevers, Disc. Faraday Soc. 25, 29 (1958).

31. D. Sec, T. Porenta, M. Ravnik and S. Zumer, Soft Matter 8, 11982-11988 (2012).

32. A. C. North and A. Rich, Nature 191, 1242-1245 (1961).

33. W. C. Earnshaw and S. C. Harrison, Nature 268, 598-602 (1977).

34. J. Dubochet, J. M. Adrian, J. J. Chang, J. C. Homo, J. Lepault, J. A. W. McDowall and P. Schultz, Quarterly Reviews of Biophysics 21, 129-228 (1988).

35. M. E. Cerritelli, N. Q. Cheng, A. H. Rosenberg, C. E. McPherson, F. P. Booy and A. C. Steven, Cell 91, 271-280 (1997).

36. J. E. Johnson and W. Chiu, Current Opinion In Structural Biology 17, 237-243 (2007).

37. J. Lepault, J. J. Dubochet, J. W. Baschong and E. Kellenberger, EMBO Journal 6, 1507-1512 (1987).

38. F. P. Booy, F. P. W. W. Newcomb, B. L. Trus, J. C. Brown, T. S. Baker and A. C. Steven, Cell 64, 1007-1015 (1991).

39. A. Leforestier and F. Livolant, Journal of Molecular Biology 396, 384-395 (2010).

40. E. Raspaud, T. Forth, C. Sao-Jose, P. Tavares and M. de Frutos, Biophysical Journal 93, 3999-4005 (2007). 
41. N. Chiaruttini, M. de Frutos, E. Augarde, P. Boulanger, L. Letellier and V. Viasnoff, Biophysical Journal 99, 447-455 (2010).

42. P. Grayson, L. Han, T. Winther and R. Phillips, Proc. Natl. Acad. Sci. USA 104, 14652-14657 (2007).

43. S. Mangenot, M. Hochrein, J. Radler and L. Letellier, Current Biology 15, 430-435 (2005).

44. N. Chiaruttini, Master's thesis, Universit Paris-Diderot - Paris VII (2010).

45. I. Ali and D. Marenduzzo, The Journal of Chemical Physics 135, 095101 (2011).

46. D. Wu, D. Van Valen, Q. C. Hu and R. Phillips, Biophysical Journal 99, 1101-1109 (2010).

47. K. J. Hanhijarvi, G. Ziedaite, M. K. Pietila, E. Haeggstrom and D. H. Bamford, Biophysical Journal 104, 2264-2272 (2013).

48. G. S. Hayward and M. G. Smith, Journal of Molecular Biology 63, 383-95 (1972).

49. H. R. Warner and D. J. McCorquodale, Bacteriophage T5 and Related Phages, Vol. 1, Springer, US (1988).

50. J. Abelson and C. A. Thomas Jr., Journal of Molecular Biology 18, 262-288 (1966).

51. A. Evilevitch, L. Lavelle, C. M. Knobler, E. Raspaud and W. M. Gelbart, Proc. Natl. Acad. Sci. USA 100, 9292-9295 (2003).

52. A. Leforestier, S. Brasiles, M. de Frutos, E. Raspaud, L. Letellier, P. Tavares and F. Livolant, Journal of Molecular Biology 384, 730-739 (2008).

53. M. de Frutos, L. Letellier and E. Raspaud, Biophysical Journal 88, 1364-1370 (2005).

54. A. Leforestier, A. Siber, F. Livolant and R. Podgornik, Biophysical Journal 100, 22092216 (2011).

55. T. Odijk, Biophysical Journal 75, 1223-1227 (1998).

56. S. Tzlil, J. T. Kindt, W. M. Gelbart and A. Ben-Shaul, Biophysical Journal 84, 16161627 (2003).

57. P. K. Purohit, J. Kondev and R. Phillips, Journal of the Mechanics and Physics of Solids 51, 2239-2257 (2003).

58. V. I. Kottadiel, V. B. Rao and Y. R. Chemla, Proc. Natl. Acad. Sci. USA 109, 2000020005 (2012).

59. A. S. Petrov and S. C. Harvey, Journal of Structural Biology 174, 137-146 (2011).

60. A. Matsuyama and M. Yano, Journal of the Physical Society of Japan 81, 034802 (2012).

61. J. P. Mahalik, B. Hildebrandt and M. Muthukumar, Journal of Biological Physics 39, 229-245 (2013).

62. I. Ali, D. Marenduzzo and J. M. Yeomans, Biophysical Journal 94, 4159-4164 (2008).

63. D. N. Fuller, D. M. Raymer, J. P. Rickgauer, R. M. Robertson, C. E. Catalano, D. L. Anderson, S. Grimes and D. E. Smith, Journal of Molecular Biology 373, 1113-1122 (2007).

64. A. S. Petrov and S. C. Harvey, Structure 15, 21-27 (2007).

65. T. E. Cloutier and J. Widom, Molecular Cell 14, 355-362 (2004).

66. T. E. Cloutier and J. Widom, Proc. Natl. Acad. Sci. USA 102, 3645-3650 (2005).

67. S. A. Harris, C. A. Laughton and T. B. Liverpool, Nucleic Acids Research 36, 21-29 (2008).

68. J. S. Mitchell, C. A. Laughton and S. A. Harris, Nucleic Acids Research 39, 3928-3938 (2011).

69. D. Demurtas, A. Amzallag, E. J. Rawdon, J. H. Maddocks, J. Dubochet and A. Stasiak, Nucleic Acids Research 37, 2882-2893 (2009).

70. J. M. Fogg, G. L. Randall, B. M. Pettitt, D. L. Sumners, S. A. Harris and L. Zechiedrich, Quarterly Reviews of Biophysics 45, 257-299 (2012). 
71. A. Leforestier and F. Livolant, Proc. Natl. Acad. Sci. USA 106, 9157-9162 (2009).

72. A. A. Kornyshev, Physical Chemistry Chemical Physics 12, 12352-12378 (2010).

73. M. Kleman, Journal de Physique 46, 1193-1203 (1985).

74. A. A. C. C. Pais, M. G. Miguel, P. Linse and B. Lindman, Journal of Chemical Physics 117, 1385-1394 (2002).

75. A. Cacciuto and E. Luijten, Phys. Rev. Lett. 96, 238104 (2006).

76. A. Al Lawati, I. Ali and M. Al Barwani, Plos One 8, e52958 (2013).

77. H. H. McAdams and A. Arkin, Proc. Natl. Acad. Sci. USA 94, 814-819 (1997).

78. D. Fraser and M. Kaern, Molecular Microbiology 71, 1333-1340 (2009).

79. E. Chapman-McQuiston and X. L. Wu, Biophysical Journal 94, 4525-4536 (2008).

80. J. J. Dennehy and I. N. Wang, BMC Microbiology 11, 174 (2011).

81. R. Gallet, T. Lenormand and I. N. Wang, Evolution 66, 3485-3494 (2012).

82. A. Arkin, J. Ross and H. H. McAdams, Genetics 149, 1633-1648 (1998).

83. L. Y. Zeng, S. O. Skinner, C. H. Zong, J. Sippy, M. Feiss and I. Golding, Cell 141, 682-691 (2010). 